\title{
Angels Santa, La Literatura popular francesa: follettines y melodramas
}

Sant M. Carmen Figuerola

\section{(2) OpenEdition}

1 Journals

\section{Édition électronique}

URL : http://journals.openedition.org/studifrancesi/475

DOI : 10.4000/studifrancesi.475

ISSN : 2427-5856

Éditeur

Rosenberg \& Sellier

\section{Édition imprimée}

Date de publication : 1 avril 2015

Pagination : 154-155

ISSN : 0039-2944

\section{Référence électronique}

Sant M. Carmen Figuerola, "Angels Santa, La Literatura popular francesa: follettines y melodramas », Studi Francesi [En ligne], 175 (LIX | I) | 2015, mis en ligne le 01 avril 2015, consulté le 18 septembre 2020. URL : http://journals.openedition.org/studifrancesi/475 ; DOI : https://doi.org/10.4000/ studifrancesi.475

Ce document a été généré automatiquement le 18 septembre 2020.

\section{(c) $($ ) $\odot \odot$}

Studi Francesi è distribuita con Licenza Creative Commons Attribuzione - Non commerciale - Non opere derivate 4.0 Internazionale. 


\title{
Angels Santa, La Literatura popular francesa: follettines y melodramas
}

\author{
Sant M. Carmen Figuerola
}

\section{RÉFÉRENCE}

ANGELS SANTA, La Literatura popular francesa: follettines y melodramas, Lleida, Pagès editors i Universitat de Lleida, 2012, «El Fil d'Ariadna», pp. 196.

1 De nos jours, il n'est plus possible d'affirmer que la littérature populaire constitue un domaine peu traité par la critique littéraire. Depuis les années ' 80 ont vu le jour de nombreux travaux sur l'écriture "populaire" ainsi que sur l'industrie culturelle, héritière en grande partie des procédures ayant caractérisé son ancêtre. Des analyses de Jean-Claude Vareille, de Michel Nathan, jusqu'aux initiatives entamées par l'Association internationale des Chercheurs en Littératures Populaires et Cultures Médiatiques présidée par Jacques Migozzi, maints spécialistes prestigieux - Charles Grivel, Jean-Yves Mollier, Daniel Couégnas, Paul Bleton, pour s'en tenir à quelques-uns - ont focalisé leur attention sur ce genre, s'il peut être ainsi dénommé. L'Espagne compte aussi une certaine tradition dans ce type d'études: Ana González, Antonio Altarriba, Elena Real, Lola Jiménez et Ángels Santa sont des noms réputés pour ceux qui s'intéressent au sujet. Malgré cet apogée, le label du populaire relève d'un corpus large et divers où il reste encore à creuser. Son expérience dans ce champ de recherche permet à l'auteur de présenter ici une synthèse rigoureuse à la fois qu'agréable à la lecture.

2 Suivant l'esprit de divulgation qui anime la collection «El Fil d'Ariadna», le volume d'Àngels Santa prend pour point de départ l'établissement d'une définition du roman populaire qui, d'après une approche englobante, concentre les différentes positions critiques depuis lesquelles il a été envisagé et, en même temps, résume les traits qui le caractérisent. Elle porte ensuite son regard sur deux manifestations: le feuilleton français et le mélodrame. Ayant une visée didactique, elle détaille les constantes 
distinctives de chaque sous-genre, considère les multiples modalités de leurs formes (roman maritime, roman frénétique, roman historique dans le cas du feuilleton...), sans pour autant oublier leurs antécédents (la tragédie et les feuilletons par rapport au mélodrame). Dans une perspective systématique, l'ouvrage passe en revue les circonstances sociologiques ayant favorisé le succès de ces produits: la progressive diffusion de la presse dans le lectorat de la France du XIXe et l'essor de l'image comme moyen publicitaire deviennent des éléments susceptibles d'expliquer l'extension du feuilleton, phénomène qui, de manière paradoxale, s'accompagne d'une rare réception critique. Quant au mélodrame, l'analyse tient aussi compte des aspects spécifiques de la vie théâtrale: sont déclinés les avatars des salles parisiennes, de ses protagonistes - les acteurs - et des éléments matériels indispensables à la représentation - les décors ou la musique - dont le but est de mettre en relief le message de l'écrivain bien que par d'autres moyens.

3 Pourtant, ces deux manifestations littéraires sont loin de représenter des entités monolithiques immuables tel qu'il appert dans le volume au moment où il présente les différentes étapes marquant l'évolution des deux sous-genres. Dans ce sens se justifie l'allusion aux auteurs les plus représentatifs: Eugène Sue, Paul Féval, Alexandre Dumas, parmi les plus connus, voisinent avec d'autres tels que Xavier de Montepin, Émile Richebourg, Pierre Decourcelle ou Charles Merouvel pour ce qui est du feuilleton; Pixérécourt, Louis Caigniez, Adolphe Dennery deviennent, à leur tour, les protagonistes du chapitre consacré au mélodrame. Pour chacun d'eux, Àngels Santa note les aspects biographiques saillants et souligne dans un deuxième temps les apports les plus novateurs dans le domaine. Malgré les limitations d'un volume à volonté divulgatrice, la spécialiste esquive les poncifs de l'histoire littéraire et fournit une contribution personnelle consistant à réfléchir sur l'intérêt de ces ouvrages chez le lecteur ou pour le public actuel. Pour améliorer l'appréhension, un index nominal contient la biographie littéraire des écrivains cités au cœur de l'analyse. De même, un glossaire de termes critiques et un tableau des événements socio-politiques et culturels ont la vertu de guider les moins initiés, soulignant le lien entre ce type de littérature et les productions littéraires «canoniques». Outre un style clair qui facilite la lecture du livre, l'auteur parsème le texte de nombreuses illustrations signifiantes puisque, d'après ses thèses, l'image a exercé une fonction essentielle au sein de cette industrie culturelle.

Un autre chapitre compile des textes critiques, dans le but de renforcer les arguments théoriques formulés: la pensée d'Antoine Court, de Charles Grivel, de Jules Janin, de Jean-Marie Thomasseau s'exprime en écho pour compléter les thèses de ce volume. D'autres voix résonnent aussi pour ramener le lecteur à la création originelle puisque des fragments d'œuvres les plus significatives figurent dans le dernier chapitre. Le lecteur peut savourer des échantillons des Trois Mousquetaires, des Mystères de Paris, de Philippe Derblay ou du Bossu. En complément du volume, la bibliographie fournit des références intéressantes, mais surtout son mérite consiste à recenser un grand nombre de numéros de revues axés sur le sujet et dont la répercussion se dilue lorsque, habituellement, ces articles sont cités de manière individuelle.

Bref, ceux qui s'intéressent au roman populaire trouveront dans cette édition espagnole un document soigneux où l'équilibre entre ses parties réussit à offrir une vue d'ensemble rigoureuse sans pour autant oublier que le succès de ce type d'écriture se doit à la soif de lecture. C'est pourquoi les morceaux choisis ont une valeur essentielle: celle d'apporter une invitation à la même, de proposer un délicieux apéritif susceptible 
de révéler l'appétence du lecteur... Phénomène d'autant plus compréhensible que pour apprécier la qualité d'un texte, il est préférable - au moins au premier abord - de s'imbiber de cet élixir magique qu'est la littérature. 